\title{
Transanal total mesorectal excision (TaTME): tips and tricks of a new surgical technique
}

\author{
Elisa Cassinotti ${ }^{1}$, Giorgio Palazzini ${ }^{2}$, Massimiliano Della Porta $^{3}$, Ilaria Grosso ${ }^{1}$, Luigi Boni ${ }^{1}$ \\ ${ }^{1}$ Department of General and Emergency Surgery at the IRCCS, Ca' Granda, Policlinico Hospital, University of Milan, Milano, Italy; ${ }^{2}$ Sapienza \\ Università di Roma Dipartimento Scienze Chirurgiche, Roma, Italy; ${ }^{3}$ Chirurgia Generale, Ospedale San Carlo, Milano, Italy \\ Contributions: (I) Conception and design: L Boni, E Cassinotti; (II) Administrative support: I Grosso; (III) Provision of study materials or patients: L \\ Boni, 1 Grosso, M Della Porta; (IV) Collection and assembly of data: L Boni, G Palazzini; (V) Data analysis and interpretation: L Boni, E Cassinotti, \\ G Palazzini; (VI) Manuscript writing: All authors; (VII) Final approval of manuscript: All authors. \\ Correspondence to: Luigi Boni. Department of General and Emergency Surgery at the IRCCS, Ca' Granda, Policlinico Hospital, University of Milan, \\ via Francesco Sforza 20122, Milan, Italy. Email: luigi.boni@unimi.it.
}

\begin{abstract}
Laparoscopic total mesorectal excision (TME) can be technically challenging especially in obese and patients with narrow pelvis. Transanal TME (TaTME) is a technique that combines abdominal and transanal endoscopic approach to overcome limitations and facilitate standard laparoscopic TME. The authors present some specific tips and tricks to perform this complex procedure using a double team approach and a new reusable transanal port.
\end{abstract}

Keywords: Rectal cancer; transanal total mesorectal excision (TaTME)

Received: 20 April 2017; Accepted: 12 May 2017; Published: 12 July 2017.

doi: 10.21037/ales.2017.05.07

View this article at: http://dx.doi.org/10.21037/ales.2017.05.07

\section{Introduction}

To date total mesorectal excision (TME), based on dissection of the presacral plane and the removal of intact mesorectum, represents the gold standard treatment for rectal cancer. The widespread adoption of this technique resulted in decreasing local recurrences and improving survival.

Laparoscopic TME has shown oncological outcomes similar to open TME but leading to the "typical" benefits of laparoscopy such as better postoperative recovery, lower morbidity and shorter hospital stay (1).

Both with open or laparoscopic approach, the main challenge is to complete TME in low rectal lesions through a sphincter saving procedure with negative distal margins, good quality specimens and reduced morbidity. Performing a good TME, especially in case of narrow pelvis, male patients, previous irradiation, advanced tumors and obesity, might be challenging (2).

Transanal TME (TaTME) is a technique that combines abdominal and transanal endoscopic approach to overcome limitations and facilitate standard laparoscopic TME.

So far multiple case series on TaTME have been reported showing feasibility and safety of the technique and promising good results in terms of post-operative and oncological outcomes (3). However, this procedure is complex, with several steps and it requires experienced surgeons aware of anatomical and technical aspects regarding this procedure (3-6).

\section{Patient selection and preoperative workout}

Preoperative patients' workout does not differ from standard laparoscopic TME, including digital pelvic examination, preoperative chest and abdominal CT scan and pelvic MRI and/or EUS. Indications for stage-based neoadjuvant chemo-radiotherapy do not change.

TaTME it is the ideal approach for patients with mid or low rectal cancer (within $8 \mathrm{~cm}$ from the anal verge), especially in males (because of narrow pelvis), obese or with bulky tumors. 


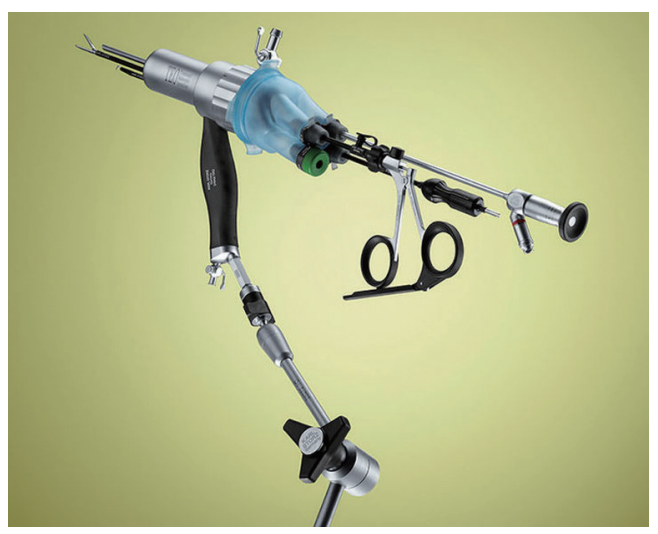

Figure $1 \mathrm{TEO}^{\circledR}$ Platform with Flexible Working Attachment and High-Flow Adaptor-B-PORT (Karl Storz GmbH \& Co, Tuttlingen, Germany).

\section{Pre-operative preparation}

Full mechanical bowel preparation is needed in order to facilitate the initial part of the transanal part of the procedure. Prophylactic antibiotics are given at induction according to hospital policy.

\section{Equipment and operative room settings}

The optimal setting to perform TaTME and to ensure safety and efficiency during both abdominal and transanal part is to arrange two laparoscopic towers, each with its own insufflator, camera and monitor. This could be very helpful both in the "two-team" simultaneous approach and in single team approach, in order not to change all operating room settings in the middle of procedure and especially to be able to control intra-abdominally when transanal excision reaches peritoneal cavity.

In our experience synchronous approach to the abdomen and to transanal dissection (two team approach) has several advantages once compared with the "single team", sequential approach: it allows to save operative time, facilitate the TME by guiding the transrectal team during the final part of the transanal dissection. Nevertheless the "two team approach" requires at least four operators preferably two scrub nurses and a complete double equipment and two separated table for instruments.

It is extremely important that all the OR personnel, the anesthesiology and surgical teams are aware of the different steps of the procedure and every possible intraoperative complication.
Patients are placed in lithotomy position and the use of suspended monitors allows a better ergonomic positioning for both teams (7).

The abdominal part of the procedure is performed as in standard laparoscopic anterior resection and it does not require dedicated instruments other that the ones preferred by the surgeon, while for the transanal part of TaTME specific devices are required $(8,9)$.

A self-fixing anal retractor (Lone Star CooperSurgical, Trumbull, CT, USA) allows good exposure of the anal canal and it is usually placed at the beginning of the transanal part of the procedure. The anal retractor is extremely useful in case of low rectal tumors when hand-sewn purse-string to close the lumen of the rectum is performed as well as after specimen removal to achieve a better exposure of the rectal stump for the anastomosis.

To perform transanal TME specific platforms and devices are needed. Some authors report the use of platforms, such as GelPOINT Path (Applied Medical, Rancho Santa Margarita, CA, USA) or SILSport (Covidien, Minneapolis, MN,USA) that represent adaptation or modification of disposable single port devices that are usually fixed to patient's perineal area, allow good instrument's triangulation, but being disposable leads to a significant increase of costs (5).

In our experience, since we started performing TaTME with a large experience in Transanal Endoscopic Microsurgery (TEM), the use of reusable system may be cost-effective and also give very fix and stable field due to the rectoscope structure.

We currently use the $\mathrm{TEO}^{\circledR}$ Platform with Flexible Working Attachment and High-Flow Adaptor-B-PORT (Karl Storz GmbH \& Co, Tuttlingen, Germany) equipped with flexible cap that allows freedom of movements and wide choice of ports (from 3 to $15 \mathrm{~mm}$ ) (Figure 1).

TaTME requires a stable field of dissection and good smoke evacuation to optimize and enhance the identification of anatomical planes and structures during the transanal dissection. Conventional insufflators designed for laparoscopic surgery have a major limitation related to the intermittent modality with which they establish and maintain the abdominal pressure.

This modality is efficient in a large cavity such as the abdomen but it is inadequate for the rectum where the volume to be insufflated and distended is limited and requires frequent smoke evacuation resulting in continuous movement of the surgical field that make the transanal 


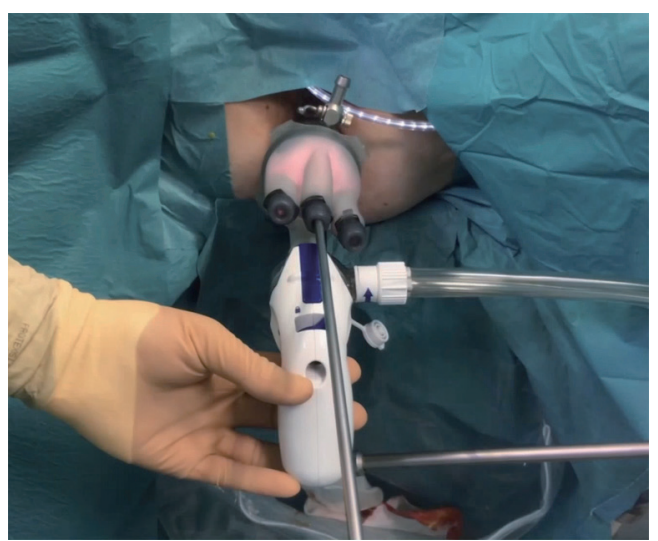

Figure 2 Continuous $\mathrm{CO}_{2}$ insufflation device inserted in transanal port.

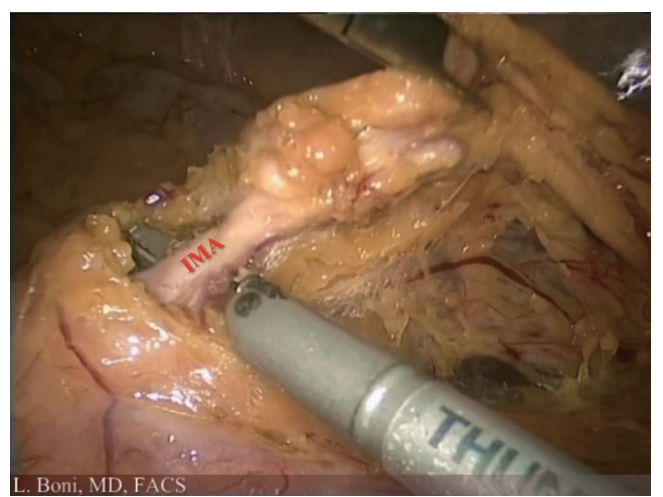

Figure 3 Laparoscopic high ligation of inferior mesenteric artery.

dissection difficult. The use of continuous $\mathrm{CO}_{2}$ insufflation device integrated with smoke evacuation system (AirSeal System Surgiquest, Milford, US) is mandatory during TaTME: it provides a real-time monitoring and maintenance of set pressure, effective smoke evacuation and cleaner camera setting (10) (Figure 2).

All the remaining device for the transanal part for the procedure can be selected according to surgeon's preferences although in our practice monopolar energy (hook and/or scissors) and bipolar forceps are needed, while advanced energy devices may not be necessary since the mesorectal excision will follow anatomical planes.

\section{Procedure}

Procedure usually starts with abdominal laparoscopic approach in order to evaluate peritoneal cavity (presence of adhesions, carcinomatosis, anatomical pelvic features etc).

A standard 4-port technique can be used, minimizing abdominal wall trauma using one $10 \mathrm{~mm}$ and three $5 \mathrm{~mm}$ ports, since no larger port for stapler is needed.

A standard medial to lateral approach is used to perform high ligation of inferior mesenteric artery and left colon mobilization (Figure 3).

Standard laparoscopic instrumentation for anterior rectal resection (clip applier, advanced energy device) is required, depending on surgeon's preferences. Full mobilization of the splenic flexure is usually required.

Transanal time of TaTME can start after peritoneal reflection incision from the abdomen or simultaneously to the abdominal part. A purse-string suture is placed distal to the tumor (manually under direct vision for very low tumors, endoscopically after transanal platform placement for mid and upper rectal lesions); at this point full-thickness incision of rectal wall is performed circumferentially and dissection carries on until reaching the peritoneal cavity.

Once the specimen has been removed, coloanal anastomosis is performed, either end-to-end or side-to-end with hand-sewn or stapled technique.

Most reports in literature describe creation of defunctioning loop ileostomy to protect TaTME very low anastomosis $(6-8,11)$.

\section{Tips, tricks and pitfalls}

As mentioned above, we do believe that full mobilization of splenic flexure is mandatory in order to have adequate bowel length for transanal specimen extraction and preparation of descending colon for a tension free low anastomosis.

In specific situations (early stage tumors in thin patients/ no previous abdominal surgery or benign disease that requires low rectal resections, such as RCU) it is possible to further reduce trauma, performing SILS abdominal access from right flank and afterwards use this incision for temporary diverting ileostomy, if needed (12).

In the abdominal part peritoneal reflection is opened circumferentially. It is recommended to enter and dissect upper mesorectal plane posteriorly along the sacral plane and laterally, respecting hypogastric nerves and ureters while anteriorly the peritoneal reflection has only to be incised, without proceeding with further dissection.

In female patients, to better expose the anterior part of mesorectum, it is possible to suspend the uterus with a percutaneous stitch. Similarly, in male patients with very 


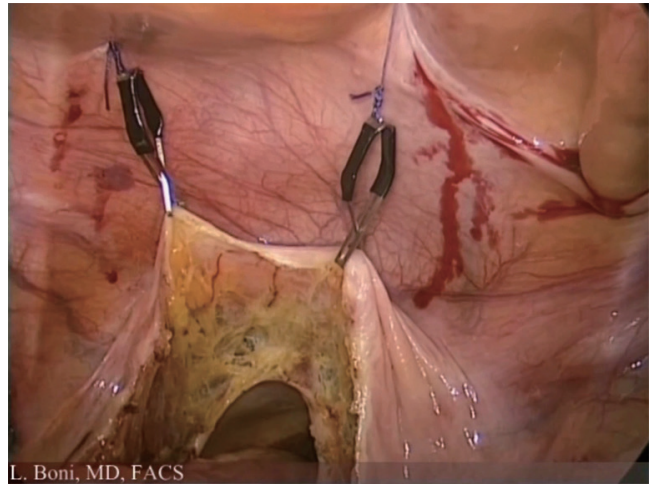

Figure 4 Peritoneal reflection lifted intra-abdominal retractor (VersaLifter, Surgical Perspectives, Strasbourg, France).

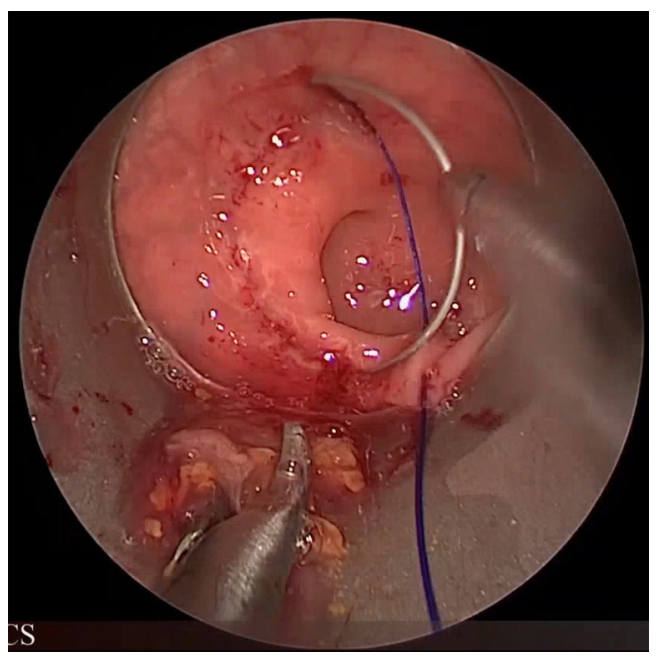

Figure 5 Rectal purse-string.

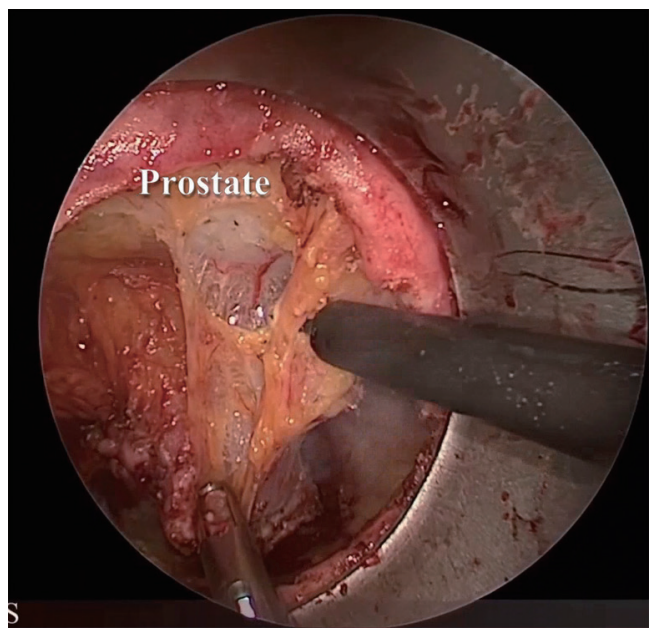

Figure 6 Antero-lateral mesorectal dissection in male patient. narrow pelvis, the anterior peritoneal reflection can be lifted up with stitches or dedicated intra-abdominal retractor and suspension systems (VersaLifter, Surgical Perspectives, Strasbourg, France) (Figure 4).

Once ligature of the inferior mesenteric vessels is performed and the descending colon mobilized, we recommend to close temporarily sigmoid colon by means of a long forceps, in order to avoid excessive $\mathrm{CO}_{2}$ insufflation into the bowel during endoscopic purse-string. Preventing excessive bowel dilation may be extremely useful if later during procedure it is necessary to work again into the abdomen (for instance for further colonic mobilization or for ileostomy creation).

During the transanal part of the procedure, it is important that rectal purse-string is full thickness and well tight. The surgeon should place "small bites" in order to obtain an air tight closure of the rectum that will allow a stable working field without blowing the colon above (Figure 5).

During the first step of the mesorectal dissection from below, the surgeon should "mark" circumferentially the mucosa of the rectum with monopolar hook and then start full thickness rectotomy usually in the posterior plane (where the plane between the presacral fascia and mesorectal envelope is relatively easy to identify) and then circumferentially.

During the initial part of the rectotomy, the surgeon will be able to appreciate the different layers of the rectal wall that have to be dissected completely to enter the mesorectal plane.

As mentioned, the dissection should continue circumferentially, carefully avoiding any asymmetrical rectal retraction that can make difficult the identification of correct plane. In male patients anterolateral dissection proceeds toward the level of prostate-seminal junction, identifying small vessels penetrating the prostate (neurovascular bundles of Walsh) that work as a landmark to find the correct plane of dissection entering Denonvilliers' fascia (Figure 6).

The same plane is to be found in females where the anterior plane of dissection should be carried out at the level of the junction of lower and middle third of posterior vaginal wall.

Excessive bleeding during mesorectal excision could reveal a wrong dissection plane, both in the anterior and posterior part of the rectum, where bleeding from the sacral venous plexus is a potential major intraoperative complication. 


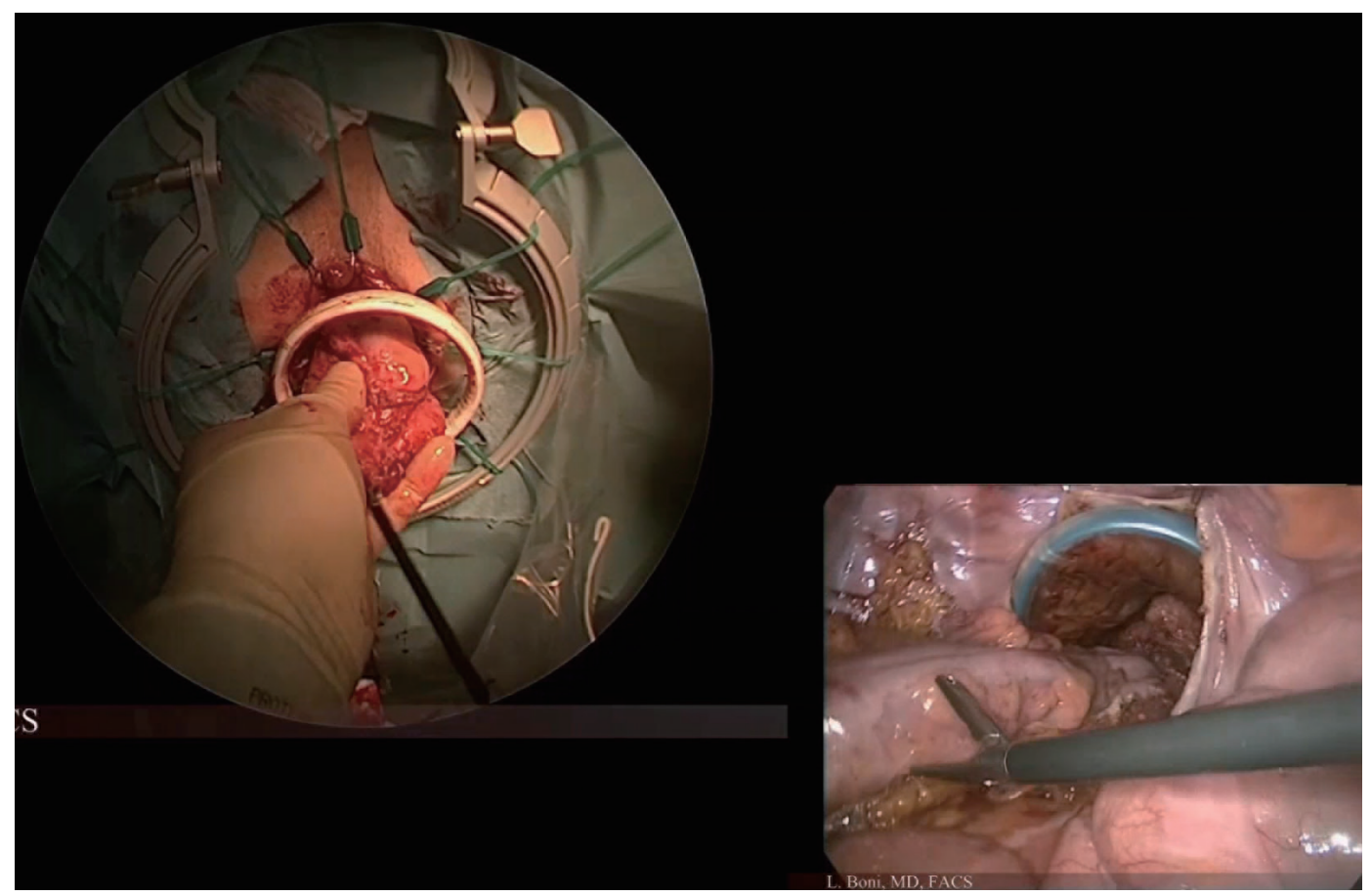

Figure 7 Transanal specimen extraction.

Especially in male patients, excessive bleeding the anterior dissection might be a sign of violation of the prostatic capsule that should be respected, also trying to avoid potential urethral injuries that have been reported in the literature (13-15).

Once TME is completed, specimen could be extracted transanally (minimizing abdominal wall trauma); however during this maneuver the surgeon must be careful not to stretch excessively the specimen, in order not to damage bowel blood supply. In case of tumors or thick mesorectum/ mesocolon, we suggest to perform sovrapubic Pfannestiel incision for specimen extraction and proximal anastomosis preparation. Wound or anal canal protection is mandatory during the extraction of the specimen (Figure 7).

Different techniques have been described for the anastomosis. Mechanical side-to-end or end-to-end anastomosis should be preferred if distal rectal stump is long enough to allow full thickness purse-string closure around the anvil of the stapler. Although any type of surgical stapler can be used for the anastomosis, it is mainly described the use of compact straight stapler (Chex ${ }^{\mathrm{TM}}$ CS COMPACT ${ }^{\mathrm{TM}}$, Frankenman International Ltd, Hong Kong) or stapler with long central rod on the anvil that allows connection between anvil and staple device before closure of pursestring $\left(\mathrm{EEA}^{\mathrm{TM}}\right.$ Hemorrhoid and Prolapse Stapler Set with
DST Series ${ }^{\mathrm{TM}}$ Technology, Medtronic, Dublin, Ireland). Nevertheless similar results can be achieved using standard circular stapler with a drainage tube connected to the anvil and drag into the anal purse-string (Figure 8).

In case of very short rectal stump, a traditional handsewn coloanal anastomosis can be performed with one or two layers suture incorporating the anorectal cuff mucosa, a portion of upper internal sphincter and full thickness muscular layer of the colon.

Choice of the technique may be influenced by surgeon experienced and should be "tailored" depending on patient's anatomy and distance of the tumor from the anorectal junction (16).

Perfusion of the bowel stumps is one of risk factors for anastomotic leakage and coloanal anastomoses, due to anatomical reason, are particularly at risk (17).

We regularly check bowel perfusion prior and, transanally, after the anastomosis to reduce the risk of post-operative leaks. Based on our experience, we suggest to perform intraoperative fluorescence angiography for colorectal microperfusion assessment; fluorescence guided surgery to evaluate anastomotic site perfusion can influence surgical decision and modify operative strategy, leading to lower leak rate and improved postoperative outcome $(18,19)$ (Figure 9). 


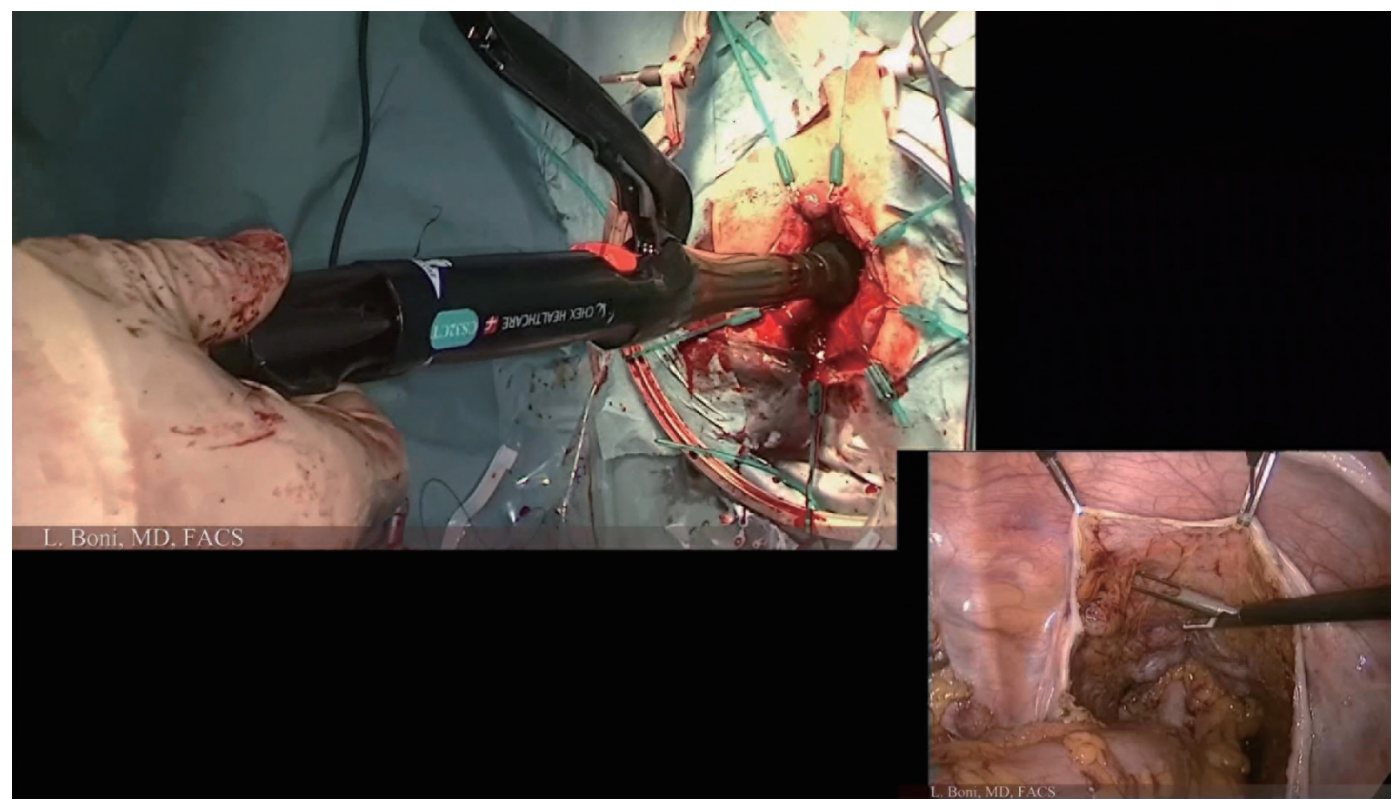

Figure 8 Mechanical coloanal anastomosis.

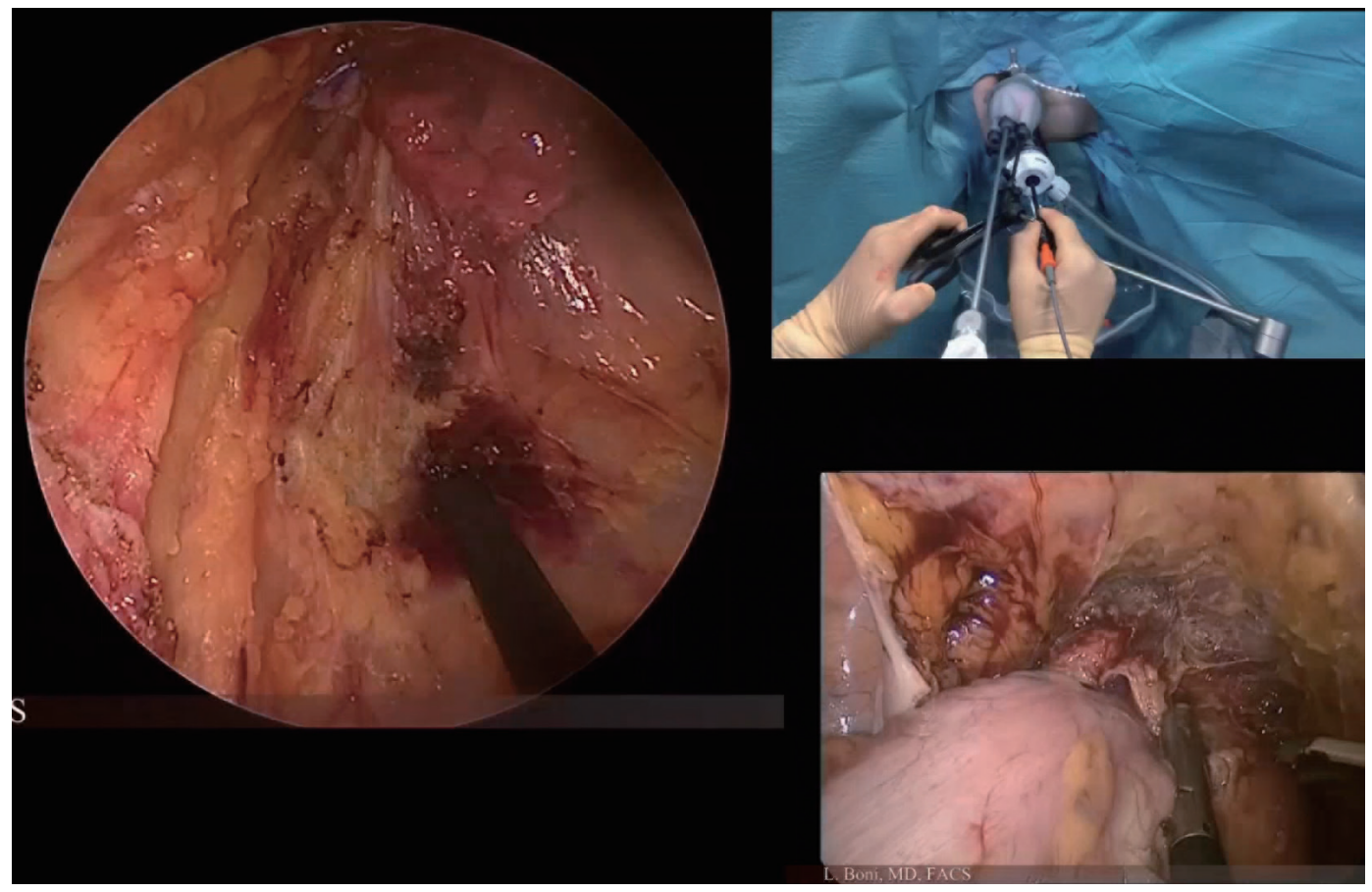

Figure 9 Intraoperative fluorescence angiography for colorectal microperfusion assessment.

Another trick that we apply, based on our experience and supported by some large studies in literature, is to place a transanal tube at the end of procedure; this aims to reduce endoluminal pressure in the anastomotic bowel portion which may be an important factor in preventing anastomotic leakage (20) (Figure 10). 


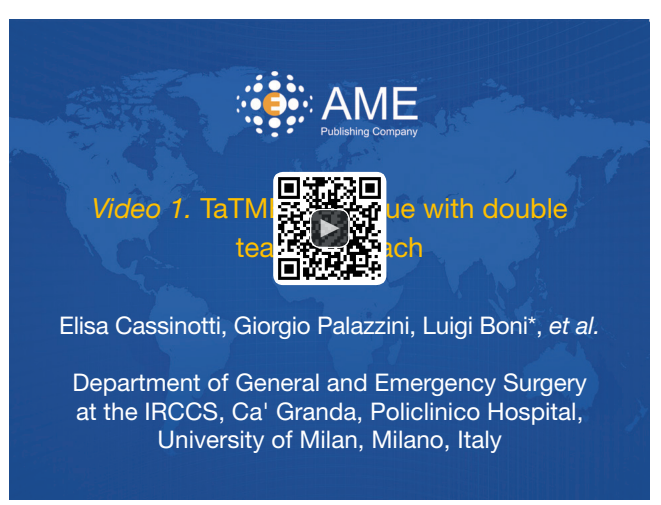

Figure 10 TaTME technique with double team approach (21). TaTME, transanal total mesorectal excision.

Available online: http://www.asvide.com/articles/1581

\section{Conclusions}

TaTME offers a combined laparoscopic and transanal approach to achieve a safe and oncologically complete TME dissection for low rectal tumors. Nevertheless it is a technically demanding and challenging procedure that requires experience in laparoscopic colorectal surgery and special attention to technical details and instrumentations.

\section{Acknowledgements}

None.

\section{Footnote}

Conflicts of Interest: The authors have no conflicts of interest to declare.

\section{References}

1. Bonjer HJ, Deijen CL, Abis GA, et al. COLOR II: A randomized trial of laparoscopic versus open surgery for rectal cancer. N Engl J Med 2015;372:1324-32.

2. Poon JT, Law WL. Laparoscopic resection for rectal cancer: a review. Ann Surg Oncol 2009;16:3038-47.

3. Araujo SE, Crawshaw B, Mendes CR, et al. Transanal total mesorectal excision: a systematic review of the experimental and clinical evidence. Tech Coloproctol 2015;19:69-82.

4. Velthuis S, Nieuwenhuis DH, Ruijter TE, et al. Transanal versus traditional laparoscopic total mesorectal excision for rectal carcinoma. Surg Endosc 2014;28:3494-9.
5. Wolthuis AM, Bislenghi G, de Buck van Overstraeten A, et al. Transanal total mesorectal excision: Towards standardization of technique. World J Gastroenterol 2015;21:12686-95.

6. Knol JJ, D'Hondt M, Souverijns G, et al. Transanal endoscopic total mesorectal excision: technical aspects of approaching the mesorectal plane from below--a preliminary report. Tech Coloproctol 2015;19:221-9.

7. Arroyave MC, DeLacy FB, Lacy AM. Transanal total mesorectal excision (TaTME) for rectal cancer: Step by step description of the surgical technique for a two-teams approach. Eur J Surg Oncol 2017;43:502-5.

8. McLemore EC, Harnsberger CR, Broderick RC, et al. Transanal total mesorectal excision (taTME) for rectal cancer: a training pathway. Surg Endosc 2016;30:4130-5.

9. Atallah S, Albert M, DeBeche-Adams T, et al. Transanal minimally invasive surgery for total mesorectal excision (TAMIS-TME): a stepwise description of the surgical technique with video demonstration. Tech Coloproctol 2013;17:321-5.

10. Nicholson G, Knol J, Houben B, et al. Optimal dissection for transanal total mesorectal excision using modified $\mathrm{CO} 2$ insufflation and smoke extraction. Colorectal Dis 2015;17:O265-7.

11. Rouanet P, Mourregot A, Azar CC, et al. Transanal endoscopic proctectomy: an innovative procedure for difficult resection of rectal tumors in men with narrow pelvis. Dis Colon Rectum 2013;56:408-15.

12. Tuech JJ, Karoui M, Lelong B, et al. A step toward NOTES total mesorectal excision for rectal cancer: endoscopic transanal proctectomy. Ann Surg 2015;261:228-33.

13. Knol J, Chadi SA. Transanal total mesorectal excision: technical aspects of approaching the mesorectal plane from below. Minim Invasive Ther Allied Technol 2016;25:257-70.

14. Atallah S, Albert M, Monson JR. Critical concepts and important anatomic landmarks encountered during transanal total mesorectal excision (TaTME): toward the mastery of a new operation for rectal cancer surgery. Tech Coloproctol 2016;20:483-94.

15. Bernardi MP, Bloemendaal AL, Albert M, et al. Transanal total mesorectal excision: dissection tips using ' $O$ 's and 'triangles'. Tech Coloproctol 2016;20:775-8.

16. Penna M, Knol JJ, Tuynman JB, et al. Four anastomotic techniques following transanal total mesorectal excision (TaTME). Tech Coloproctol 2016;20:185-91. 
17. Jafari MD, Wexner SD, Martz JE, et al. Perfusion assessment in laparoscopic left-sided/anterior resection (PILLAR II): a multi-institutional study. J Am Coll Surg 2015;220:82-92.e1.

18. Boni L, David G, Dionigi G, et al. Indocyanine greenenhanced fluorescence to assess bowel perfusion during laparoscopic colorectal resection. Surg Endosc 2016;30:2736-42.

19. Boni L, Fingerhut A, Marzorati A. et al. Indocyanine green

doi: 10.21037/ales.2017.05.07

Cite this article as: Cassinotti E, Palazzini G, Della Porta M, Grosso I, Boni L. Transanal total mesorectal excision (TaTME): tips and tricks of a new surgical technique. Ann Laparosc Endosc Surg 2017;2:111. fluorescence angiography during laparoscopic low anterior resection: results of a case-matched study. Surg Endosc 2017;31:1836-40.

20. Wang S, Zhang Z, Liu M, et al. Efficacy of transanal tube placement after anterior resection for rectal cancer: a systematic review. World J Surg Oncol 2016;14:92.

21. Cassinotti E, Palazzini G, Della Porta M, et al. TaTME technique with double team approach. Asvide 2017;4:272. Available online: http://www.asvide.com/articles/1581 